\title{
Visual Acuity and Contrast Sensitivity with compressed motion video
}

\author{
Piet Bijl \& Sjoerd C. de Vries \\ TNO Defense, Security \& Safety: Human Factors, P.O. Box 23, Soesterberg, The Netherlands \\ Phone: +31346356 368, fax: +31346353977 \\ E-mail: piet.bijl@tno.nl, sjoerd.devries@tno.nl
}

\begin{abstract}
Video of Visual Acuity (VA) and Contrast Sensitivity (CS) test charts in a complex background was recorded using a CCD camera mounted on a computer-controlled tripod and fed into real-time MPEG2 compression/decompression equipment. The test charts were based on the Triangle Orientation Discrimination (TOD) test method and contained triangle test patterns of different sizes and contrasts in four possible orientations. In a perception experiment, VA and CS thresholds at the $75 \%$ correct level were obtained for three camera velocities $(0,1.0$ and $2.0 \mathrm{deg} / \mathrm{s}$ or $0,4.1$ and 8.1 pix/frame) and four compression rates (no compression, $4 \mathrm{Mb} / \mathrm{s}, 2 \mathrm{Mb} / \mathrm{s}$ and $1 \mathrm{Mb} / \mathrm{s}$ ). VA is shown to be rather robust to any combination of motion and compression. CS however dramatically decreases when motion is combined with high compression ratios. The data suggest that with the MPEG2 algorithm the emphasis is on the preservation of image detail at the cost of contrast loss.
\end{abstract}

Keywords: sensor, image compression, performance, TOD, Acuity, Contrast Sensitivity Target Acquisition

\section{INTRODUCTION}

The amount of digitally stored and transmitted image data is rapidly growing due to the increasing use of digital cameras and an ever growing pixel resolution. Image compression techniques are necessary to deal with the increasing data volume. Current popular compression standards are JPEG for static pictures and MPEG-2, MPEG-4 and MJPEG2000 ${ }^{1}$ for motion video, but every day new schemes are being proposed that claim to outperform the current standards ${ }^{2}$. Of course, one of the parameters of interest is compression ratio. Reductions of the required storage capacity or network bandwidth by a factor of 50 to 100 are feasible. A high compression ratio, however, comes at the cost of information loss and the introduction of artifacts in the resulting image. Well-known artifacts of jpeg-compression for instance are ringing in text or near sharp edges, and blockiness in smooth parts of the image.

Several methods have been proposed to develop perceptually optimal quantization schemes and minimize the visibility of artifacts, for example based on the human visual system's contrast sensitivity and masking properties ${ }^{3,4}$. In addition, metrics have been developed to quantify the observer's opinion on the quality of a degraded image ${ }^{5,6,7}$.

Aside from esthetical aspects, compression may reduce performance on the tasks that operators need to perform on the imagery. Studies on performance loss due to compression are sparse. Recently, O'Shea \& Jacobs ${ }^{8}$ measured the effect of JPEG and JPEG2000 compression with different rates on vehicle identification. The findings in their study were that JPEG2000 with moderate compression ratios (30:1) only slightly reduces identification range $(-10 \%)$, while a similar compression rate with JPEG reduces range by $50 \%$. Higher compression ratios $(50: 1)$ seriously degrade performance for both techniques. Since the experiment was performed on a complex target set and the amount of conditions was limited (still images, high contrast targets only), it is difficult to relate the degradation to certain factors in the compression techniques.

In the present study we measure task performance on compressed imagery with different compression ratios but deviate from O'Shea \& Jacobs in two ways. First, we perform the test with two standard tasks from the TOD methodology': Visual Acuity and Contrast Sensitivity (see Chapter 2 for an overview) in order to find out which one is mostly affected by the compression. Second, we use motion video with MPEG-2 compression and record both static and dynamic scenes with different rotation speeds of the camera. Since performance is expected to depend on background complexity, we decided to avoid a clinical uniform laboratory environment and performed the test out in the field using test charts against a complex forest/urban background.

Infrared Imaging Systems: Design, Analysis, Modeling, and Testing XX, edited by Gerald C. Holst Proc. of SPIE Vol. 7300, 730006 · C 2009 SPIE · CCC code: 0277-786X/09/\$18 · doi: 10.1117/12.819948 
This paper is organized as follows. Chapter 2 provides a short overview of the TOD methodology to characterize sensor performance and to calculate range predictions in the field. The experimental method is described in Chapter 3. Results are given in Chapter 4, and an example range prediction with compressed video is calculated in Chapter 5. The results are discussed in Chapter 6.

\section{TOD SENSOR PERFORMANCE CHARACTERIZATION}

The Triangle Orientation Discrimination ${ }^{9}$ (TOD) method is a way to quantify sensor performance with a human-in-theloop. The method provides a sensor performance measure (the TOD curve, see section 2.1) and a model to calculate Target Acquisition (TA) performance in the field (the TOD TA model, see section 2.2).

\subsection{TOD SENSOR PERFORMANCE CURVE}

\subsubsection{Laboratory assessment}

The TOD is a sensor system specific curve (or set of curves) that describes the ability of a human observer using the sensor under test to discriminate between equilateral triangles of four possible orientations (apex Up, Down, Right or Left, see Figure 1). Independent variables are triangle angular size $S$ (defined as the square-root of the triangle area, in $\mathrm{mrad}$ ), triangle contrast (its definition depends on the spectral range on the sensor: thermal contrast $\Delta T=T_{\text {target }}-T_{\text {background }}$ for thermal imagers or visual contrast $C=\left|L_{\text {target }}-L_{\text {background }}\right| / L_{\text {background }}$ for visual or NIR devices) and background luminance $L_{\text {background }}$ for visual or NIR devices. A detailed description of the assessment procedure is given elsewhere ${ }^{10}$.

During the test, triangle patterns of different size and contrast on a uniform background are presented to the observer who has to indicate their orientation even if he is not sure. Threshold is defined at the $75 \%$ correct level and is obtained by fitting a Weibull function through the data. Image degradations induced by the sensor (such as blur, noise and sampling) make the judgments more difficult and shift the $75 \%$ correct thresholds towards a larger test pattern size $S$ or to a higher contrast. In this way, the method evaluates the combined effect of all image degradations within the sensor system including the observer.

The method yields a threshold curve or set of curves of contrast versus reciprocal size $S^{-1}$ (in $\mathrm{mrad}^{-1}$ ). See Figure $2 \mathrm{a}$ for an example with a CCD camera system. Using reciprocal angular size is convenient for several reasons: i) a higher value means higher acuity or better performance, ii) range is proportional this value, iii) the effects of atmospheric loss on performance in the field are easily included in the TOD plots, and iv) the curves are directly comparable to the more conventional MRTD and MRC.
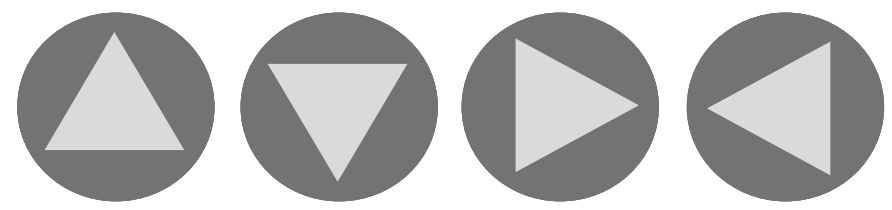

Figure 1 The test pattern or stimulus in the TOD method is an equilateral triangle with one of four possible orientations: apex Up, Down, Left or Right. The observer has to indicate its orientation. Task difficulty depends on test pattern size and contrast. From Bijl \&Valeton?

Visual Acuity (VA), Contrast Sensitivity (CS) and Luminance Sensitivity (LS) are special points on the TOD curves. VA (in $\left.\mathrm{mrad}^{-1}\right)$ is defined as the cut-off reciprocal triangle size at high contrast $(\mathrm{C}=100 \%$ or $\Delta T=2 \mathrm{~K})$. CS is defined as the reciprocal of the contrast threshold for a very large test pattern (triangle angular size $S=32 * \mathrm{VA}^{-1}$ ). LS (for visual or NIR devices) is the background luminance at which a large high contrast triangle is at threshold (i.e. the lowest luminance where anything can be seen with the device and VA and CS merge), see Figure 2b.

Recently ${ }^{11,12}$ the method has been extended to be able to quantify the effects of background non-uniformity. This is of particular interest with automatic gain control and/or targets that are present in dark areas of the scene and may be used to quantify the benefits of signal processing techniques such as Local Area Contrast Enhancement. 


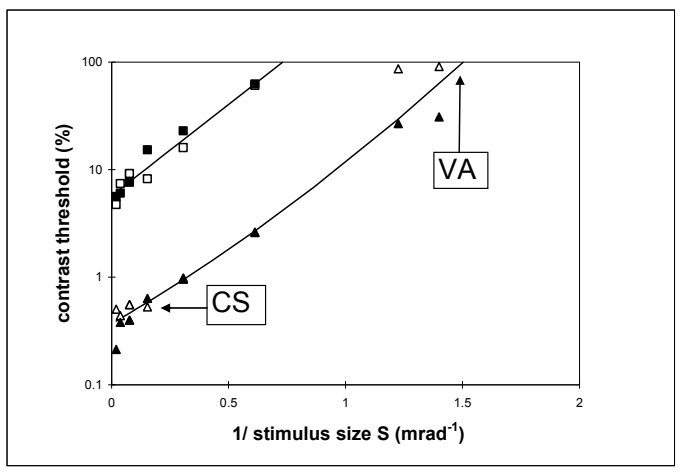

(a)

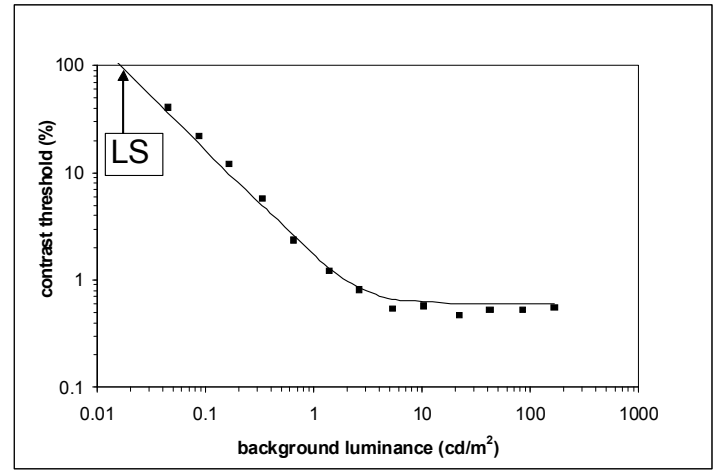

(b)

Figure 2 (a) TOD curves for a typical CCD camera system. Plotted are $75 \%$ correct contrast thresholds $C_{t h r}$ as a function of reciprocal triangle size $S^{-1}$ (in $\mathrm{mrad}^{-1}$ ) at two different light levels: $\mathrm{L}=165 \mathrm{~cd} / \mathrm{m}^{2}$ (triangle symbols) and $\mathrm{L}=0.33 \mathrm{~cd} / \mathrm{m}^{2}$ (square symbols) and best fit polynomials. (b) $C_{t h r}$ for a large triangle $\left(S=32 \cdot \mathrm{VA}^{-}\right.$ ${ }^{1}$ ) as a function of background luminance $L_{\text {background }}$. VA is defined as the cut-off reciprocal triangle size at high contrast $(\mathrm{C}=100 \%)$, and $\mathrm{CS}$ as the reciprocal contrast threshold for a large triangle: $S=32 \cdot \mathrm{VA}^{-1}$. LS is defined as the luminance level at which at which a large high contrast triangle is at threshold. From Bijl \&Valeton?

\subsubsection{Simulation model}

The TOD for a (real or simulated) sensor can be calculated using an image based simulation consisting of three modules ${ }^{13,14}$ : i) a triangle test pattern generator, ii) a sensor simulation module and iii) a Human Visual System (HVS) module. Test patterns are degraded by the simulated sensor and presented to the biologically plausible HVS module that judges their most likely orientation. Thresholds are obtained with a Monte Carlo technique.

Recently ${ }^{15}$ the development of a software framework EO-VISTA (Electro-Optical VISualization and TA tool) was started that enables communication with other simulation modules such as scene, atmosphere, image enhancement or other sensor simulation models ${ }^{16,17}$. Currently, a connection is made with EOSTAR $^{18}$ scene + atmosphere model. Combinations with real components are possible as well. This combined visual simulation of the chain from scene to observer and TA model provides a huge amount of operational and R\&D applications including automated measurement, sensor design, scene visualization and/or tactical decision aids ${ }^{15}$.

\subsubsection{Analytical approximation}

Aside from the simulation model (section 2.1.2), a simple analytical expression was developed to quickly predict performance as a function of sampling and blur properties of the sensor ${ }^{19}$.

\subsection{TOD FIELD RANGE PREDICTION}

Field range prediction with the TOD TA model is extremely simple and based upon the ACQUIRE ${ }^{20}$ range performance model, in which the original MRTD/MRC curves, the Johnson criteria $N_{50}$ (for identification, recognition and detection) and the Target Transfer Probability Functions (TTPF's) are replaced by the TOD, a target set/acquisition level specific magnification factor $M_{75}$ and a Weibull function, respectively.

Mathematics and details are provided in the Appendix. An example calculation is shown in Chapter 5.

\subsection{APPLICATIONS AND VALIDATION}

The TOD laboratory method is simple, intuitive, accurate, easy-to-perform, avoids observer bias, includes statistical significance tests, and can be performed both in the lab and in the field ${ }^{11,21}$.

It can be applied to any well-sampled or under-sampled image forming sensor system. Test equipment has been developed to characterize sensors from different spectral ranges such as visual ${ }^{22}$, thermal ${ }^{23}$ and X-ray ${ }^{24}$ and effects of 
motion and image enhancement techniques have been quantified empirically $y^{21,25,26,27,28}$. The method has also been applied to quantify the effects of image enhancement on automated systems and has been shown to outperform MSE (Mean Square Error) methods ${ }^{29}$. Finally, the method is applied to quantify the static and dynamic visual performance of simulators ${ }^{30}$. The method is recommended by the $\mathrm{ITU}^{7}$ for videophony display characterization. The HVS model (2.1.2) enables automated measurement and automatic characterization of image enhancement methods ${ }^{13,14,30}$.

The TOD Target Acquisition model has been used to predict ship identification and recognition from CCD imagery as a function of contrast and aspect angle ${ }^{31}$, military vehicle identification with well- and undersampled thermal imagers ${ }^{32}$ and identification of static and moving two-hand held objects from thermal imagery ${ }^{33}$. Despite the simple principles of both the test method and the TA model, the predictions with respect to sampling effects, target contrast, target orientation, and motion are very satisfying. See also section 2.4 for a comparison with alternative approaches.

Note that prediction of search is not included in the TOD model. The visual search process is radically different from the identification process and is better predicted by other approaches ${ }^{34}$.

\subsection{CORRESPONDENCE WITH OTHER APPROACHES}

The two most promising alternative approaches to the TOD are the TTP (Targeting Task Performance) metric ${ }^{35,36,37}$ and the MTDP test method in combination with the TRM3 range performance model ${ }^{38,39}$.

The TTP metric ${ }^{35,36,37}$ is a strictly theoretical metric and has no corresponding simple camera test. Available software are the NVThermIP thermal imager model and a corresponding suite for visual and NIR devices. Similar to the TOD TA model, task complexity is characterized by a parameter: $V_{50}$. The model, however, is much more complex and less intuitive than the TOD. While the TOD has not changed over the years, the TTP metric is continuously being improved ${ }^{35,36,37}$ and extended, e.g. to incorporate signal processing effects. A quantitative comparison between TOD and TTP in $2002^{40}$ showed huge differences. With the newer version ${ }^{36}$ the TTP predictions are closer to the TOD although the models still weigh the effects of under-sampling differently ${ }^{41}$. In their newest version ${ }^{37}$, the aliased signal from undersampled imagers is treated as noise and the model now takes into account the effects of in-band aliasing. A first quantitative comparison ${ }^{19}$ indicates that this newest TTP matches the TOD predictions quite closely!

The Minimum Temperature Difference Perceived (MTDP) model $^{38,39}$ is an adaptation of the MRTD to make it applicable to undersampled imagers. TRM3 is the TA range model associated with the MTDP, and the model is available as a mature software package. A comparison with the two other approaches has been made in $2002^{40}$ and the model has not changed since. The laboratory test can easily be performed with a standard MRTD setup but suffers from the same well-known MRTD observer bias problems which have been overcome with the TOD method. In addition, the method is less suited to cope with dynamic system performance ${ }^{27}$.

\section{METHODS}

\subsection{CAMERA SYSTEM}

A Panasonic NV-GS-17 miniDV camcorder mounted on a computer-controlled pan\& tilt tripod was used. Resolution was 720 by 576 pixels, and Horizontal Field Of View was set at $7.1^{\circ}$ (IFOV $=0.17 \mathrm{mrad}$ ). Vibration correction (default) was switched off.

The camera signal (PAL composite video) was fed into a computer-controlled real-time MPEG-2 encoder (Tandberg DSNG Voyager E5740) and decoded using a Tandberg Receiver TT1222.

With a switch, either the direct camera signal or the encoded/decoded video signal was presented on a 14" Sony HR Trinitron PVM-14M4E color CRT (resolution 800 tv-lines). 


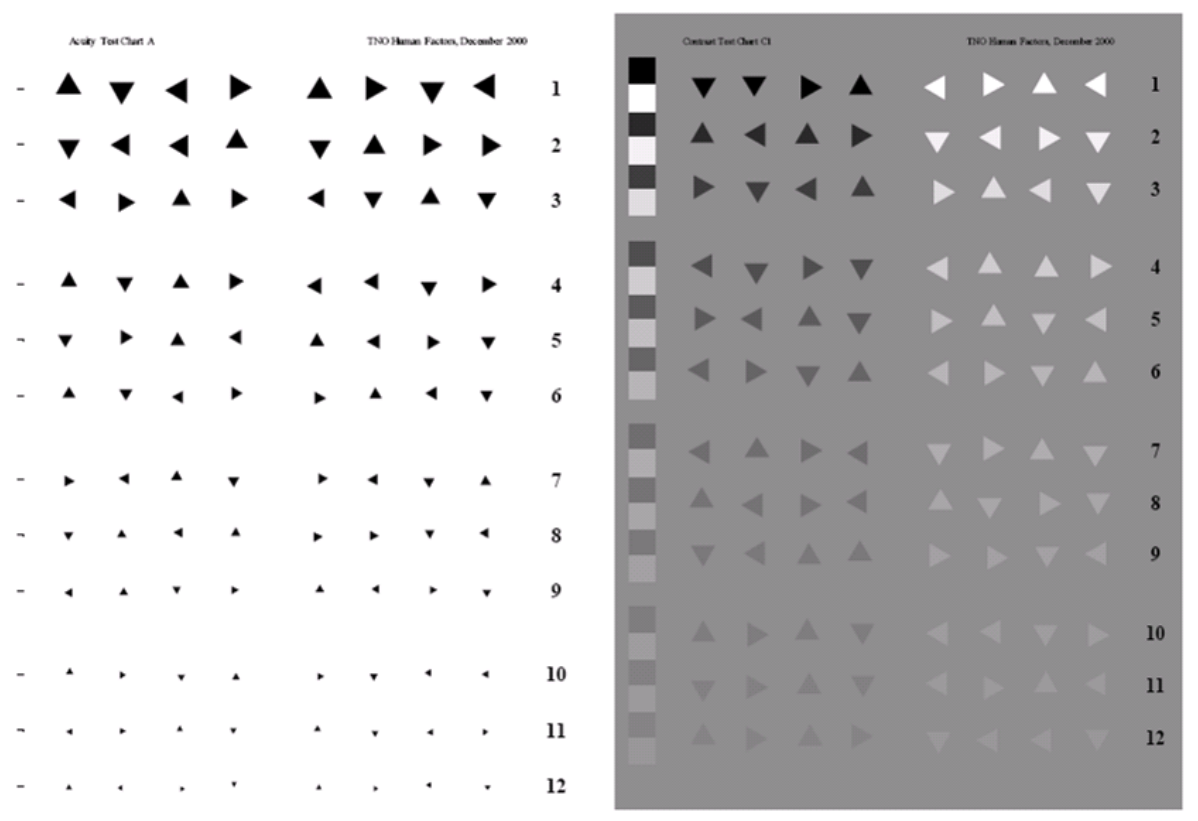

Figure 3. TOD VA (Visual Acuity) and CS (Contrast Sensitivity) test charts. From Hogervorst \& Bij1 ${ }^{22}$.

\subsection{TEST SETUP AND MEASUREMENT PROCEDURE}

The measurements were carried out using TOD VA (Visual Acuity) and CS (Contrast Sensitivity) test charts (see Figure 3). Standard chart size is A3 (297 x $420 \mathrm{~mm})$. A detailed description of the procedure is provided elsewhere ${ }^{22}$.

With the VA chart, test pattern contrast is approximately $90 \%$. Triangle size on the standard chart varies from $S=8 \mathrm{~mm}$ (top row) to $1.75 \mathrm{~mm}$ (bottom row), but in this experiment a smaller chart (A5, a size reduction of 0.50 ) was used.

Test pattern size on the CS chart is $S=8 \mathrm{~mm}$ (triangle base $=12 \mathrm{~mm}$ ) and contrast varies from $100 \%$ (top row, average over the white and black test patterns) to approximately $3 \%$ (bottom row). These low contrasts could be obtained using a dither technique.

For each condition, the observer first indicated a row of test patterns that could be judged correctly. Then, starting from that line downwards, he had to judge the orientation of all the test patterns while the experimenter checked the result. The procedure stopped when the observer score was around guess level (25\% correct). Two observers participated in the experiment: SdV and PB. Thresholds were obtained by fitting a Weibull function through the data ${ }^{10}$ and calculating the 75\%-correct sizes (VA chart) and contrasts (CS chart). Threshold standard error is approximately 10\% (VA) - 15\% (CS). Even though the experiment was performed with a limited amount of observers and presentations, the accuracy is sufficient for the purpose of the study.

\subsection{MEASUREMENT CONDITIONS}

The VA and CS test charts were placed side-by-side against a detailed forest/urban background. Distance from the camera was $5.30 \mathrm{~m}$. An impression of the scene is shown in Figure 4.

The light and weather conditions were good. Some stray light fell on the observer display introducing some loss of contrast. This may increase the absolute contrast thresholds but can only have a second order effect on the relative thresholds.

VA and CS measurements were performed under the following conditions: 
- Compression rate: (i) no compression (approx. $270 \mathrm{Mb} / \mathrm{s}$ ), (ii) $4 \mathrm{Mb} / \mathrm{s}$, (iii) $2 \mathrm{Mb} / \mathrm{s}$ en (iv) $1 \mathrm{Mb} / \mathrm{s}$.

- Camera velocity: static, $1 \mathrm{deg} / \mathrm{s}$ (4.1 pix/frame), $2 \mathrm{deg} / \mathrm{s}$ (8.1 pix/frame). In the static condition, the test charts were in the image center. During the dynamic conditions, the camera moved back and forth in horizontal direction from 10 to +10 degrees with respect to the static condition.

The static condition with a $2 \mathrm{Mb} / \mathrm{s}$ compression rate is missing.

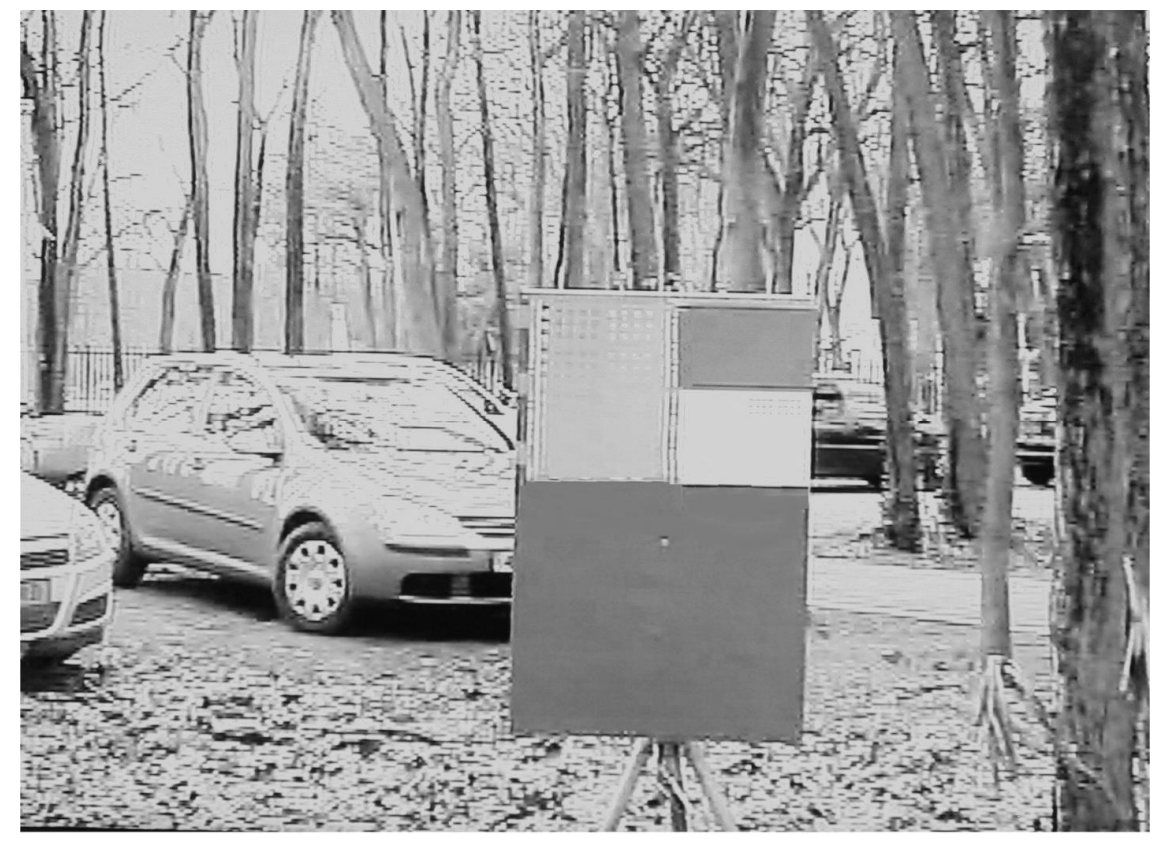

Figure 4 Scene with the two test targets

\section{RESULTS}

The results of the VA experiments are presented in Figure 5a, and some example images are shown in Figure 6. We see that:

- The effect of compression on the acuity of the system is limited. A compression rate of $2-4 \mathrm{Mb} / \mathrm{s}$ reduces acuity by only $5 \%$. Even at the lowest bitrate and a camera velocity of $2 \mathrm{deg} / \mathrm{s}$, the loss in acuity is $25 \%$ compared to the uncompressed static condition.

- A slow motion of $1 \mathrm{deg} / \mathrm{s}$ increases the visual acuity with uncompressed video (not significant, but see the Discussion).

- For uncompressed static video with this camera system and FOV, VA $=2.5 \mathrm{mrad}^{-1}$. This value corresponds quite well to the visual acuity of the unaided human eye.

- The corresponding threshold triangle size at high contrast $S=2.3$ pixels on the CCD chip.

The results of the CS experiments are presented in Figure 5b. Some example images are shown in Figure 6. We see that:

- Contrast Sensitivity for static imagery is relatively insensitive to compression rate.

- The effect of compression on moving imagery is dramatic: while $\mathrm{CS}=14$ (contrast threshold $=7 \%$ ) for uncompressed moving scenes, it degrades to $2-3$ (contrast threshold $=30-50 \%$ ). Compared to the static situation, the loss is a factor of $10-25$.

- In the experiment, motion itself partly reduces CS because the test chart moves and is only visible for a limited time during a camera sweep 
Additional results

- When scene motion is applied to compressed video, parts of the test chart containing several rows of low-contrast test patterns completely disappear and become a uniform image

- Under less severe compression, low-contrast triangles appear as blocky patterns and their quality varies in time. Initial estimates may be much poorer than reported here but integration over time helps the observer to make a correct decision.

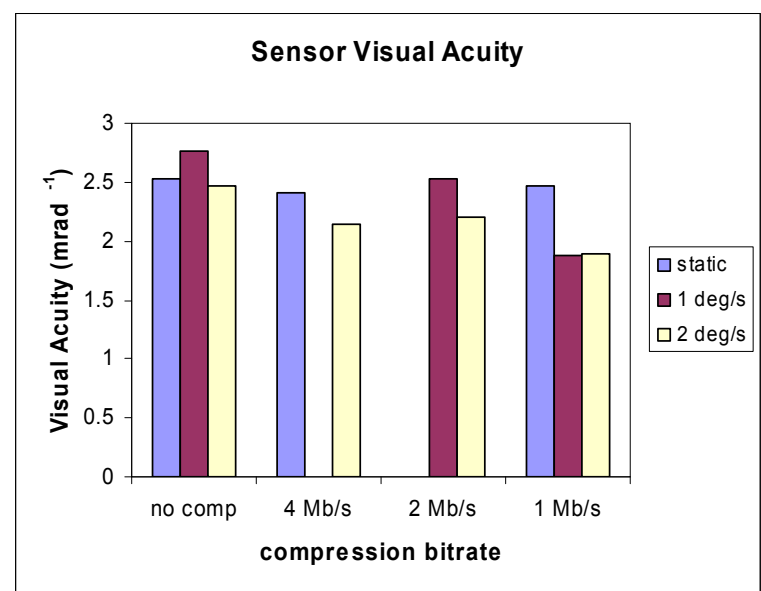

(a)

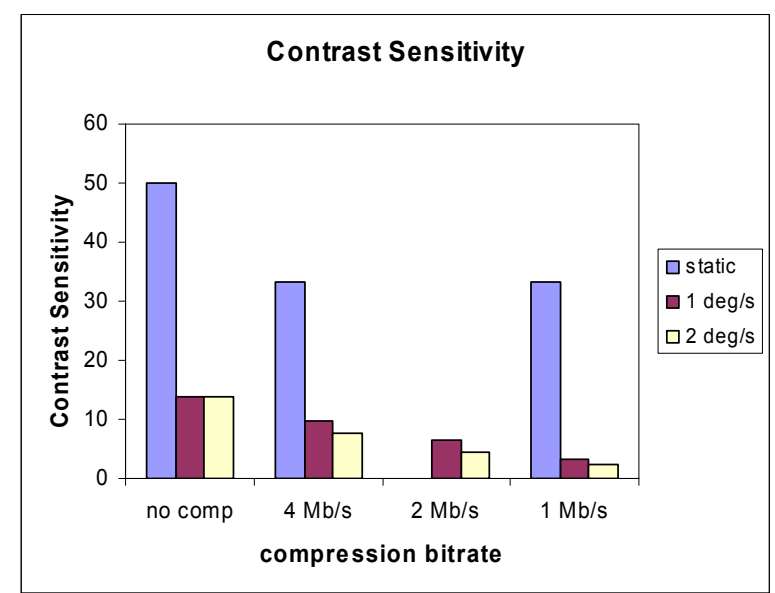

(b)

Figure 5 (a) Visual Acuity and (b) Contrast Sensitivity for the camera system with MPEG-2 compression at different compression rates and with different camera velocities. The experimental error is typically $10 \%$ (VA) and $15 \%$ (CS). The effect of compression on system VA for both static and dynamic imagery is acceptable. CS for static imagery is relatively insensitive to compression rate, but in combination with motion the effect is dramatic.

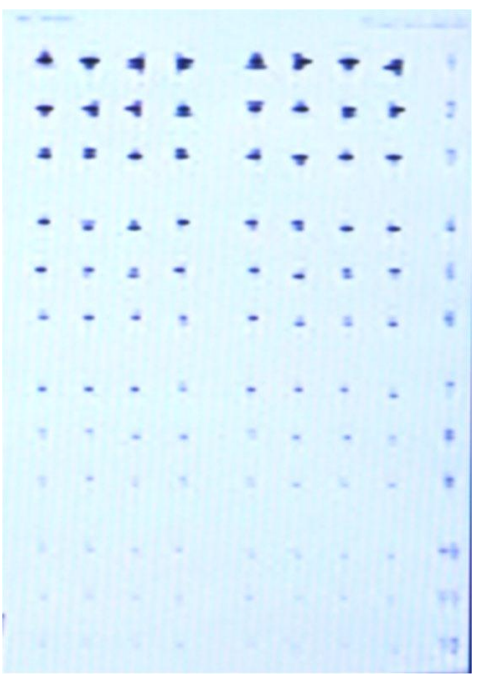

(a)

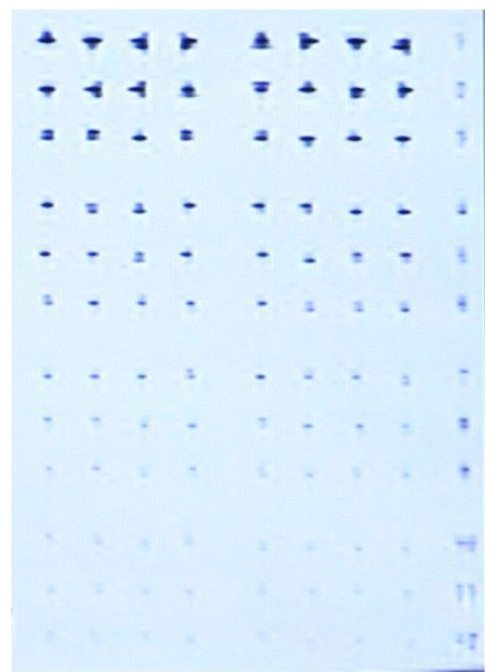

(b)

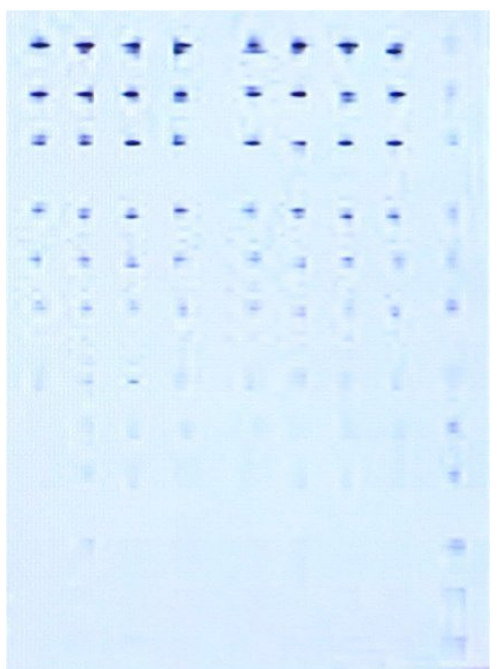

(c)

Figure 6 Image of the VA chart at (a) $4 \mathrm{Mb} / \mathrm{s}$, static, (b) $1 \mathrm{Mb} / \mathrm{s}$, static and (c) $1 \mathrm{Mb} / \mathrm{s}, 2 \mathrm{deg} / \mathrm{s}$. 


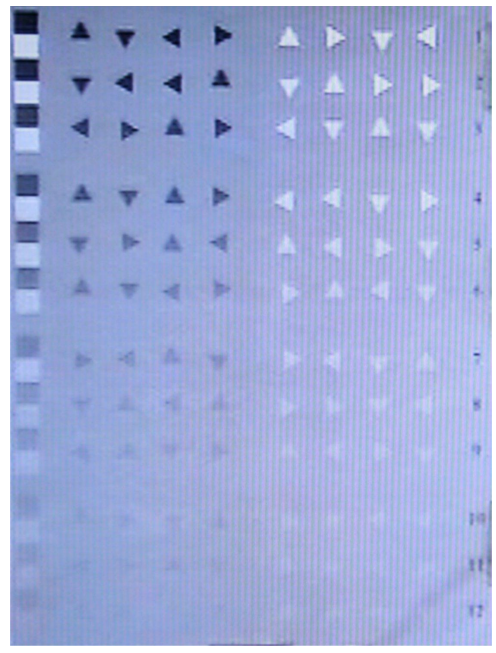

(a)

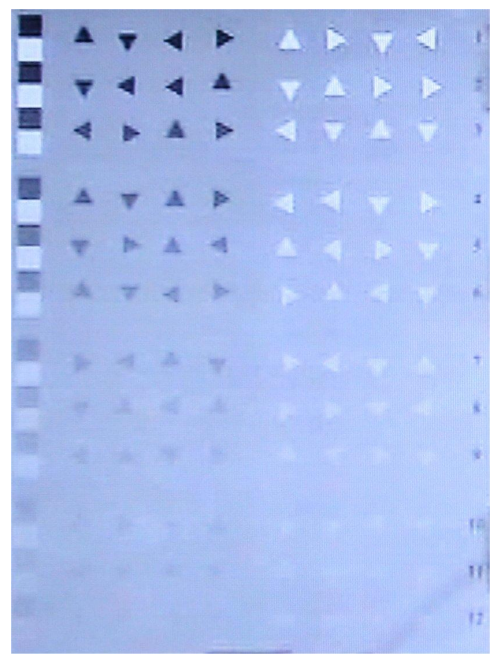

(b)

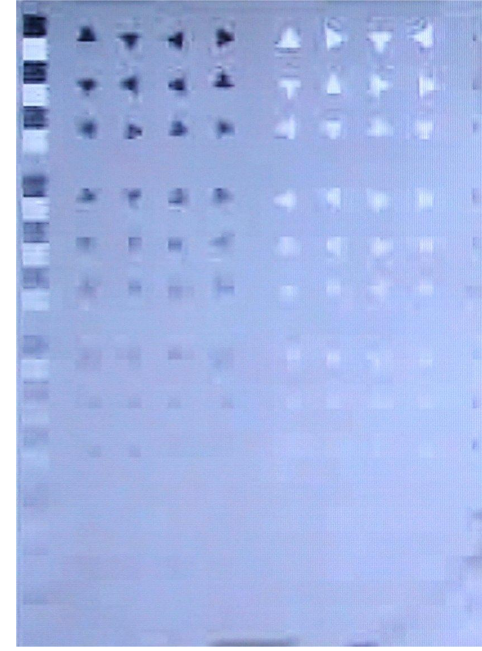

(c)

Figure 7 Image of the CS chart at (a) $4 \mathrm{Mb} / \mathrm{s}$, static, (b) $1 \mathrm{Mb} / \mathrm{s}$, static and (c) $1 \mathrm{Mb} / \mathrm{s}, 2 \mathrm{deg} / \mathrm{s}$.

\subsection{TOD ESTIMATE}

\section{FIELD RANGE PREDICTION EXAMPLE}

From the data in Figure 5 we are able to estimate TOD curves for the different conditions. For each curve, we only have two points available (VA at $100 \%$ and a contrast threshold at a fixed triangle size), but when plotted on a lin-log scale the TOD curve of most systems is approximately linear (see also Figure 2). The results for six conditions (three compression rates times two velocities) are given in Figure 8. This figure again shows the very limited effect of MPEG2 compression on sensor performance when the scene is static (continuous lines) but the huge effect in combination with motion. 


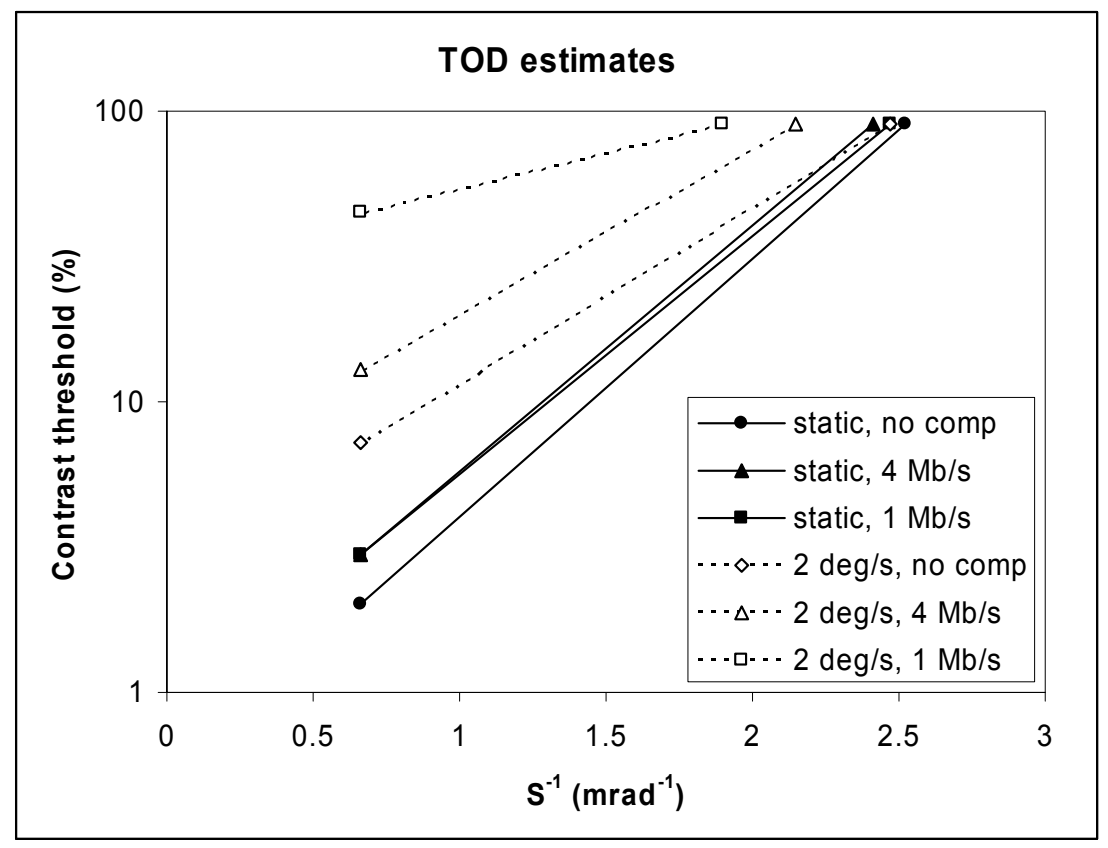

Figure 8 TOD estimates for a number of conditions from the experiment, constructed from the data in Figure 5. When the scene is static (continuous lines), sensor performance is only slightly affected by MPEG-2 compression. In combination with motion, however, the effect of compression is huge.

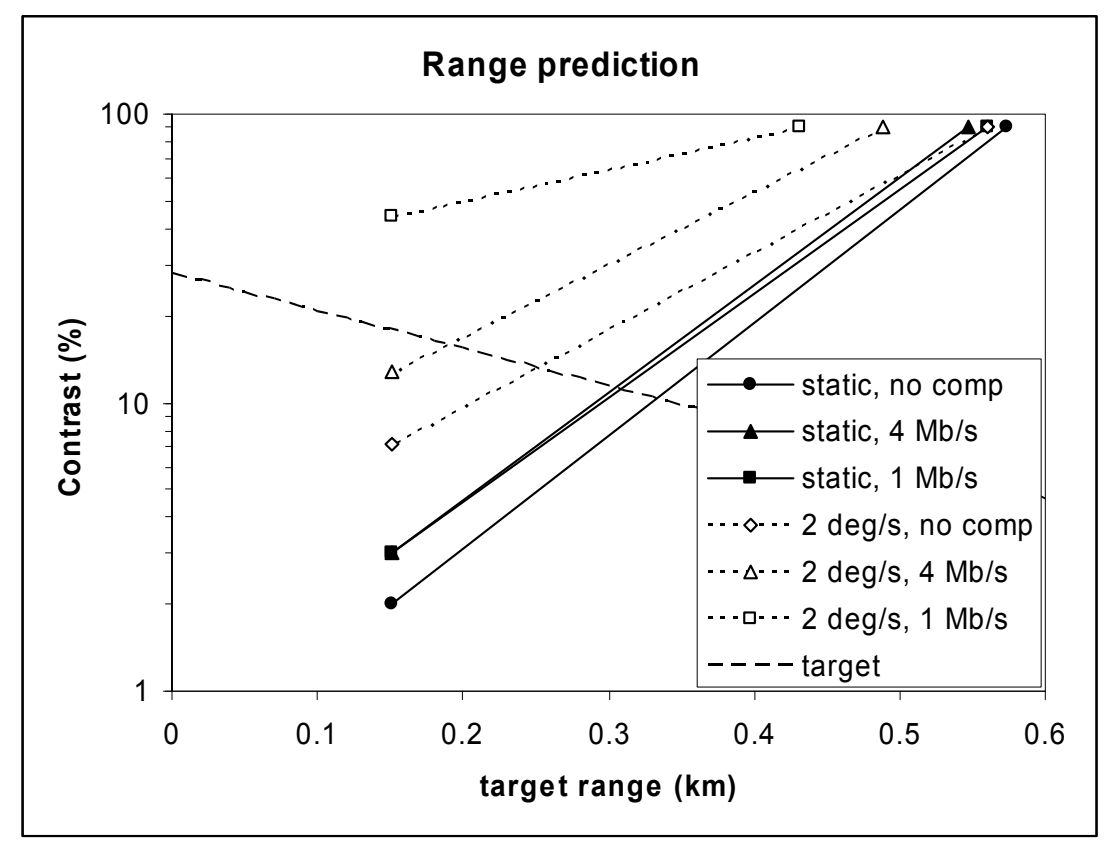

Figure 9 Identification range prediction with the sensor system for a typical military target set.

\subsection{TARGET ACQUISITION RANGE PREDICTION}


The results can be used to calculate acquisition ranges for targets in the field. The required theory is provided in the Appendix. As an example we take Tracked Armored Vehicle Identification with a visible device (required characteristic target parameters and $M_{75}$ are given in Table 1). Further, we take luminance $L=1000 \mathrm{~cd} / \mathrm{m}^{2}$ (normal day light) and visibility $V=1 \mathrm{~km}$. With equation (2) we convert the TOD curves in Figure 8 to contrast versus range curves for the military target set. See Figure 9. In addition, we plot target apparent contrast as a function of range, given the visibility $V$ (dashed line). Threshold identification range $r_{75}$ is reached where the apparent contrast line crosses the TOD curve.

For the static uncompressed condition, $r_{75}=340 \mathrm{~m}$ (Figure 9). With compressed video (4Mb/s and $\left.1 \mathrm{Mb} / \mathrm{s}\right), r_{75}=310 \mathrm{~m}$ which is just $10 \%$ lower. With motion however, range depends critically on compression rate. For the uncompressed condition, $r_{75}=260 \mathrm{~m}$, with $4 \mathrm{Mb} / \mathrm{s}$, range $r_{75}=190 \mathrm{~m}$ and with $1 \mathrm{Mb} / \mathrm{s}$ no range can be estimated since initial target contrast is below the threshold contrast measured with the TOD.

\section{DISCUSSION AND CONCLUSIONS}

Performance evaluation of encoded video is often based on a subjective observer opinion on the quality of a degraded image. This is particularly important from an esthetic point of view, but the result is not necessarily a good predictor of operator task performance with the resulting image.

In this study, we apply the TOD method ${ }^{9}$ to systematically determine the combined effect of MPEG-2 compression and motion on visual task performance. In principle, this method can be applied to assess performance loss with any video codec.

For static imagery, the effect of MPEG-2 with large compression rates on both acuity and contrast sensitivity is very limited (see Figure 8).

With a moving camera, the effect of compression rate is large (see Figure 8). Acuity is preserved quite well but the effect on contrast sensitivity is dramatic especially when the bitrate comes below $4 \mathrm{Mb} / \mathrm{s}$. The finding is in agreement with the bitrate of 4-9 Mb/s generally advised for MPEG-2 compression of SDTV video.

The combined effect of compression and motion on object identification range can be significant. With the procedure shown in Chapter 5 the maximum allowed compression rate to assure a certain task performance can be calculated.

In the image, the combination of motion and compression converts low contrast triangle test patterns on a uniform background in blocky patterns of variable quality. Integration over a period of time helps the observer to correctly judge the blocky patterns. At higher compression rates low contrast targets completely disappear and low contrast scenes may become uniform.

In Figure 5 we observe an increase of the visual acuity with uncompressed video when a slow motion of $1 \mathrm{deg} / \mathrm{s}$ is applied. Although the increase is not significant in this experiment, it has been observed in other studies as well $1^{25,27}$ and may be ascribed to the ability of a human observer to integrate information from several positions of the objects over the pixel raster.

For uncompressed static video with this camera system, Visual Acuity corresponds with a threshold triangle size $S=2.3$ pixels on the CCD chip. In other studies, often a ratio around 1.5 is found $d^{9,19}$. The difference may be ascribed to two factors: 1) in this study a color camera with Bayer filter was used, and such a system may have a reduced acuity compared to monochrome systems, and 2) the conditions for the observer were not perfect due to straylight falling on the observer display. The latter, however, should only have a limited effect on acuity because of the high contrast targets used.

\section{ACKNOWLEDGMENTS}

This study was part of a project carried out for the Dutch Ministry of Transportation (RWS). 


\section{REFERENCES}

1. D. Taubman \& M. Marcellin (2002). JPEG2000: Image Compression Fundamentals, Standards, and Practice, Kluwer, Berlin 2002.

2. F. Moore, B. Babb, M. Peterson \& G. Lamont. (2009). Evolved transforms improve image compression. SPIE Newsroom, February 2009.

3. Watson, A. B. (1993). DCT quantization matrices visually optimized for individual images. Human Vision, Visual Processing, and Digital Display IV. Rogowitz ed. 1993 SPIE.Bellingham, WA.

4. Watson, A. B. (1994). Perceptual optimization of DCT Color Quantization Matrices. Proc. IEEE International Conference on Image Processing, Austin, TX.

5. Wolf, S. \& Pinson, M. (2002). Video Quality Measurement Techniques. National Telecommunications and Information Administration Report 02-392, June 2002.

6. ITU-T Recommendation G.1070, "Opinion model for video-telephony applications," Recommendations of the ITU, Telecommunication Standardization Sector.

7. ITU-T Recommendation P.910, "Subjective video quality assessment methods for multimedia applications," Recommendations of the ITU, Telecommunication Standardization Sector.

8. P.D. O'Shea \& E.L. Jacobs (2006). Effects of image compression on sensor performance. Proc. SPIE, Vol. 6207, 62070B

9. Bijl, P.\& Valeton, J.M. (1998a). TOD, the alternative to MRTD and MRC. Optical Engineering 37, 7, 1976 - 1983.

10. Bijl, P.\& Valeton, J.M. (1999). Guidelines for accurate TOD measurement. SPIE Proceedings, Vol. 370114 - 25.

11. P. Bijl, J.W.A.M. Alferdinck, O.K. Ahmed, P.B.W. Schwering (2007). Naar een standaard beoordelingsmethodiek voor de beeldkwaliteit van cameraketens op basis van de TOD-methode. (Report DV-2007 C439). Soesterberg, The Netherlands: TNO Defence, Security and Safety (in Dutch).

12. Bijl, P. \& Vries, S.C. de, (2008).Herziening specificatie videosysteem voor de Zeeverkeerscentrale Waddenzee. [Visual requirements update for a camera system for the Sea traffic control center]. (Report DV 2008-C486). Soesterberg, The Netherlands: TNO Defence, Security and Safety (in Dutch).

13. De Lange, D.J., Valeton, J.M. \& Bijl, P. (2000). Automatic characterization of electro-optical sensors with imageprocessing, using the Triangle Orientation Discrimination (TOD) method. SPIE Proceedings, Vol. 3701, 104-111.

14. Hogervorst, M.A., Bijl, P. \& Valeton, J.M. (2001). Capturing the sampling effects: a TOD sensor performance model. SPIE Proceedings Vol. 4372, 62-73.

15. Bijl, P., Hogervorst, M.A. \& Vos, W. (2008). Modular Target Acquisition model \& visualization tool..In: Infrared Imaging Systems: Design, Analysis, Modeling, and Testing XIX, 6941, 69410E.

16. Wittenstein, W. (2006). pcSITOS User's Guide and model description. Report FGAM-FOM, Ettlingen, Germany.

17. MAVIISS (MTF-based visible and infrared imaging system simulation) software is available from JCD Publishing, 2932 Cove Trail, Winter Park, FL 32789. See www.JCDPublishing.com

18. Kunz, Gerard J.; Degache, Marianne A. C.; Moerman, Marcel M.; van Eijk, Alexander M. J.; Neele, Filip P.; DossHammel, Stephen M.; Tsintikidis, Dimitri (2004). Status and developments in EOSTAR, a model to predict IR sensor performance in the marine environment. Proceedings of the SPIE, Volume 5572, pp. 101-111.

19. Bijl, P. \& Hogervorst, M.A.(2009). Sensor performance as a function of sampling (d) and optical blur (F $\lambda$ ). SPIE Proceedings Vol. 7300-11 (in press).

20. J. A. D'Agostino et al., "ACQUIRE range performance model for target acquisition systems version 1 user's guide," U.S. CECOM Night Vision \& Electron. Sensors Directorate Rep., Fort Belvoir, VA, May 1995

21. Driggers, R.G., Krapels, K., Murrill, S., Young, S.S., Thielke, M. \& Schuler, J. (2004). Superresolution performance for undersampled imagers. Optical Engineering 44, 1.

22. Hogervorst, M.A. \& Bijl, P. (2003). Testkaarten voor meting van resolutie en contrastgevoeligheid: een gebruikershandleiding [Test charts for the measurement of resolution and contrast sensitivity: a User's guide. (Report TM-03-A005) Soesterberg, The Netherlands: TNO Human Factors (in Dutch).

23. Valeton, J.M., Bijl, P., Agterhuis, E. \& Kriekaard, S. (2000). T-CAT, a new Thermal Camera Acuity Tester. SPIE Proceedings Vol. 4030, $232-238$.

24. Bijl, P., Hogervorst, M.A., Valeton, J.M. \& Ruiter, C.J. de (2003). BAXSTER: An Image Quality Tester for X-ray Baggage Screening Systems. SPIE Proceedings Vol. 5071, 341-352.

25. Krapels, K., Driggers, R.G. \& Teaney, B. (2005). Target-acquisition performance in under-sampled infrared imagers: static imagery to motion video. Applied Optics, 44 (33), 7055-7061 
26. K. Krapels, R. G. Driggers, E. Jacobs, S. Burks, and S. Young (2007). Characteristics of infrared imaging systems that benefit from superresolution reconstruction. Appl. Opt. 46, 4594-4603 (2007)

27. Bijl, P., Schutte, K. \& Hogervorst, M.A. (2006). Applicability of TOD, MRT, DMRT and MTDP for dynamic image enhancement techniques. SPIE Proceedings 6207.

28. Dijk, J., Bijl, P. \& Bouma, H. (2009). Quantitative performance evaluation of image enhancement techniques on low-light level cameras. SPIE Proceedings Vol. 7300 (in press).

29. W. M. van Eekeren, K. Schutte, O. R. Oudegeest, and L. J. van Vliet (2007). Performance Evaluation of SuperResolution Reconstruction Methods on Real-World Data. EURASIP Journal on Advances in Signal Processing, vol. 2007, Article ID 43953.

30. Kappé, B, \& Bijl, P. (2009). Viseval 1: psychofysische metingen. Report DV-2009. Soesterberg, The Netherlands: TNO Defence, Security and Safety (in Dutch) (in preparation).

31. Bijl, P.\& Valeton, J.M. (1998b). Validation of the new TOD method and ACQUIRE model predictions using observer performance data for ship targets. Optical Engineering 37, 7, 1984 - 1994.

32. Bijl, P., Valeton, J.M. \& de Jong, A.N. (2000). TOD predicts target acquisition performance for staring and scanning thermal imagers, SPIE Proceeding Vol. 4030, 96-103.

33. Beintema, J.A., Bijl, P., Hogervorst, M.A. \& Dijk, J. (2008). Target Acquisition performance: effects of target aspect angle, dynamic imaging and signal processing. In: Infrared Imaging Systems: Design, Analysis, Modeling, and Testing XIX, 6941-11 (in press).

34. Toet, A.; Kooi, F.L.; Bijl, P.; Valeton, J.M. Visual conspicuity determines human target acquisition performance. Opt. Eng. 1998, 37 (7), 1969-1975.

35. Vollmerhausen, R., \& Driggers, R.G (1999). NVTherm: next generation night vision model. Proc. IRIS Passive Sensors, 1, 121-134.

36. Vollmerhausen, R., \& Jacobs, E. (2004). The Targeting Task Performance (TTP) Metric A New Model for Predicting Target Acquisition Performance. Report No. A394224, CNVESD, Ft. Belvoir (VA).

37. Vollmerhausen RH, Driggers RG, and Wilson DL (2008). Predicting range performance of sampled imagers by treating aliased signal as target-dependent noise. Journal of the Optical Society of America. A, Optics, image science, and vision 25(8):2055-65

38. Wittenstein, W. (1999). Minimum temperature difference perceived - a new approach to assess undersampled thermal imagers. Optical Engineering 38, 5, $773-781$.

39. Wittenstein, W., Fick, W. \& Raidt, U. (1996). Range Performance of Two Staring Imagers - Presentation of the Field Trial and Data Analysis. Proc. SPIE Conf. on Infrared Imaging Systems, 2743, 132, (1996).

40. Bijl, P., Hogervorst, M.A. \& Valeton, J.M. (2002). TOD, NVTherm and TRM3 model calculations: a comparison. SPIE Proceedings Vol. 4719, 51-62.

41. Bijl, P. \& Hogervorst, M.A.(2007). NVThermIP vs TOD: matching the Target Acquisition range criteria. SPIE Proceedings 6543, pp. 65430C.

42. Driggers et al. (2006). Current infrared target acquisition approach for military sensor design and wargaming Infrared imaging systems. Proc. SPIE, Vol. 6207, pp. 620709.1-620709.17

\section{A.1 Mathematics}

\section{APPENDIX: TOD TARGET ACQUISITION MODEL}

When the effects of atmospheric reduction are limited, probability versus range relationship $P(r)$ can be calculated with the following two equations ${ }^{41}$ :

(1) $P(r)=1-4^{-\left(r_{75} / r\right)^{\beta}}$

(2) $r_{75}=\frac{A}{S_{C_{0}} \cdot M_{75}}$

where $r_{75}$ is the target acquisition range (in $\mathrm{km}$ ) at probability $P=0.75, \beta$ determines the slope of the relationship and has value $\beta=1.25$ in our model ${ }^{41}, A$ is the target set characteristic size (in m, see section A.2), $C_{0}$ is the target set characteristic inherent RSS contrast ( $\Delta T_{0}$ for thermal, see section A.2), and $S_{C 0}$ is the corresponding triangle threshold 
size (triangle square-root area in mrad) from the TOD curve. Finally, $M_{75}$ is a magnification factor between required target and triangle size that quantifies the task difficulty (see section A.2).

With atmospheric reduction the solution is not analytical. An example calculation is provided in Chapter 5 and Figure 9. First, equation (2) is generalized to construct contrast versus range functions from the TOD at any probability level $P$ (note that these functions are scaled functions of the TOD curves as in Figure 2 with target range on the ordinate):

(3) $r_{C, P}=\frac{A}{S_{C} \cdot M_{P}}$

(4) $M_{P}=\left[\frac{\log (1-P)}{\log (0.25)}\right]^{1 / \beta} \cdot M_{75}$

Next, the relationship between apparent contrast and range for the target set is calculated:

(5) $\Delta T_{\text {apparent }}(r)=\Delta T_{0} \cdot \exp (-\sigma \cdot r)$ or $C_{\text {apparent }}(r)=C_{0} \cdot \exp \left(-\frac{r}{3 V}\right)$

where $\sigma$ is the atmospheric attenuation (in $\mathrm{km}^{-1}$ ) and $V$ is the meteorological visibility (in $\mathrm{km}$ ), i.e. the range at which the apparent contrast of a black target against the sky is reduced to 5\%. On a lin-log-scale as in Figure 2 and Figure 9, apparent contrast versus range appears as a linear line.

Finally, acquisition ranges for any probability $P$ are found at the intersections between (3) and (5) and a probabilityversus-range relationship as in equation (1) can be constructed.

\section{A.2 Target and task specific parameters}

Each target set comes with a characteristic size, defined as the average target square-root area (in $\mathrm{m}$ ) over all the targets in the set, and a characteristic root-sum-square of external and internal contrast $\triangle$ TRSS (thermal) or CRSS:

$$
\Delta T R S S=\sqrt{\left(\left(T_{t \text { arg } e t}-T_{\text {background }}\right)^{2}+\sigma_{t \text { arg } e t}^{2}\right)} \text { or } C R S S=\sqrt{\left(\left(\frac{L_{t \text { arget }}-L_{\text {background }}}{L_{\text {background }}}\right)^{2}+\sigma_{t \text { arget }}^{2}\right.}
$$

The magnification factor $M_{75}$ between target and triangle characteristic size depends on task difficulty but is sensor independent (except for sensor spectral range). It needs to be assessed once for a target $\operatorname{set}^{31,32,33}$. It has a function similar to the parameter $V_{50}$ that is used in the TTP metric (see 2.4) and has been determined for a variety of representative target sets in the visible and thermal infrared ${ }^{42}$. A simple equation ${ }^{41}$ may be used to convert one parameter into the other:

(7) $M_{75}=0.60 \cdot V_{50}$

Table 1 gives some example $M_{75}$-values derived from $V_{50}$ estimates $^{42}$, target characteristic dimensions and contrasts. 
Table $1 M_{75}$ magnification factors, characteristic dimensions and characteristic contrasts for some example target discrimination tasks in the visible and the thermal infrared, converted from $V_{50}$ estimates $^{42}$.

\begin{tabular}{|c|c|c|c|c|c|}
\hline Discrimination & Band & Object Set & $M_{75}$ & $\begin{array}{c}\text { Char Dim } \\
\mathrm{A}(\mathrm{m})\end{array}$ & RSS Contrast \\
\hline $\begin{array}{c}\text { Armored Vehicle } \\
\text { Recognition }\end{array}$ & LWIR & $\begin{array}{c}\text { Tracked } \\
\text { Wheeled Armored } \\
\text { Wheeled Soft }\end{array}$ & 10.1 & 3.0 & $3.4 \mathrm{~K}$ \\
\hline $\begin{array}{c}\text { Tracked Armored } \\
\text { Vehicle Identification }\end{array}$ & LWIR & $\begin{array}{c}\text { 2S3,BMP,M1A,M2, } \\
\text { M60,M109,M113, } \\
\text { M551,T55,T62,T72, ZSU2 }\end{array}$ & 14.0 & 3.0 & $4.7 \mathrm{~K}$ \\
\hline $\begin{array}{c}\text { Tracked Armored } \\
\text { Vehicle Identification }\end{array}$ & Visible & $\begin{array}{c}\text { 2S3,BMP,M1A,M2, } \\
\text { M60,M109,M113, } \\
\text { M551,T55,T62,T72, ZSU2 }\end{array}$ & 13.2 & 3.0 & 0.28 (unitless) \\
\hline
\end{tabular}

